\title{
ON THE IRREDUCIBLE COMPONENTS OF THE COMPACTIFIED JACOBIAN OF A RIBBON
}

\author{
MICHELE SAVARESE
}

\begin{abstract}
In this paper we study the irreducible components of the compactified Jacobian of a ribbon $X$ of arithmetic genus $g$ over a smooth curve $X_{\text {red }}$ of genus $\bar{g}$. We prove that when $g \geq 4 \bar{g}-2$ the moduli space of rank 2 semistable vector bundles over $X_{\text {red }}$ is not an irreducible component and we determine the irreducible components in which it is contained. This answers a question of D. Chen and J.L. Kass in [CK] and completes their results.
\end{abstract}

The aim of this short paper is to answer to [CK, Question 4.8], so the conventions and notations are the same of the cited place. Let us recall them.

A curve is an irreducible projective $k$-scheme of dimension 1 , where $k$ is an algebraically closed field; while a ribbon is a curve whose reduced subscheme is a smooth curve and whose nilradical is locally generated by a nonzero and square-zero element. In other words it is a primitive multiple curve of multiplicity 2 in the sense of $[\mathrm{D}]$. Observe that this definition of ribbon is more restrictive than others present in literature; e.g. in $[\mathrm{BE}, \S \mathrm{I}]$ irreducibility, projectiveness and dimension 1 are not required and the reduced subscheme is just connected.

Throughout this article $X$ will denote a ribbon and $\mathscr{N} \subset \mathscr{O}_{X}$ its nilradical. It is immediate to check that $\mathscr{N}$ can be seen as a line bundle on $X_{\text {red }}$ and that its degree on $X_{\text {red }}$ is $\operatorname{deg}(\mathscr{N})=2 \bar{g}-1-g$, where $\bar{g}$ is the genus of $X_{\text {red }}$ and $g=1-\chi\left(\mathscr{O}_{X}\right)$ is the genus of $X$.

A generalized line bundle $\mathscr{I}$ on $X$ is a pure (i.e. not having proper subsheaves of finite support) coherent sheaf whose generic stalk $\mathscr{I}_{\eta}$ is isomorphic to $\mathscr{O}_{X, \eta}$, where $\eta$ is the generic point of $X$ (in other words it is a generalized divisor in the sense of Hartshorne, because on primitive multiple curves pure is equivalent to reflexive). Its degree is $\operatorname{deg}(\mathscr{I})=\chi(\mathscr{I})-\chi\left(\mathscr{O}_{X}\right)$, while its index $b(\mathscr{I})$ is the length of the torsion part of its restriction to $X_{\text {red }}$. This is the definition of index given for any pure coherent sheaf on a ribbon in [D, §6.3.7], which is equivalent to the more involved one given in [CK, Definition 2.7], that is specific for generalized line bundles. It is easy to check that $b(\mathscr{I})$ is a non-negative integer. Moreover, it holds also that $\operatorname{deg}(\mathscr{I})-b(\mathscr{I})$ is

2010 Mathematics Subject Classification. 14D20, 14H40, 14H60.

Key words and phrases. Compactified Jacobians, Ribbons, Generalized Line Bundles, Multiple Curves. 
an even number; more precisely $\operatorname{deg}(\mathscr{I})-b(\mathscr{I})=2 \operatorname{deg}(\overline{\mathscr{I}})$, where $\overline{\mathscr{I}}$ is the unique line bundle on $X_{\text {red }}$ such that $\left.\mathscr{I}\right|_{X_{\text {red }}}=\overline{\mathscr{I}} \oplus T$ with $T$ a torsion sheaf (see [CK, Fact 2.8] and its reference; note that in the statement of the cited fact there is a typographical error: $b\left(\mathscr{I}^{\prime}\right)$ should be $\operatorname{deg}(\overline{\mathscr{I}}))$.

Chen and Kass study the moduli space $\mathrm{M}\left(\mathscr{O}_{X}, P_{d}\right)$ of semistable pure coherent sheaves of generic length 2 on $X$ of fixed degree $d$. They show that the points of $\mathrm{M}\left(\mathscr{O}_{X}, P_{d}\right)$ correspond either to $S$-equivalence classes of semistable generalized line bundles of degree $d$ or to those of direct images on $X$ of semistable rank 2 vector bundles of degree $e=d+$ $\operatorname{deg}(\mathscr{N})$ on $X_{\text {red }}$. Observe that $\mathrm{M}\left(\mathscr{O}_{X}, P_{0}\right)$ is a natural compactification of the Jacobian of $X$ when the line bundles on $X$ are stable, (i.e. when $g \geq 2 \bar{g}$, by [CK, Lemma 3.2]).

The main results about its global geometry are the following:

Fact 1.

(i) Let $j$ be an integer of the same parity of $d$ such that $0 \leq j \leq$ $-\operatorname{deg}(\mathscr{N})-1=g-2 \bar{g}$ and let $\bar{Z}_{j}$ be the closure of the locus of stable generalized line bundles of index $j$. Then $\bar{Z}_{j}$ is a $g$-dimensional irreducible component of $\mathrm{M}\left(\mathscr{O}_{X}, P_{d}\right)$ and any irreducible component containing a stable generalized line bundle is of the form $\bar{Z}_{j}$ for some $j$.

(ii) Let $\mathrm{M}(2, e)$ be the moduli space of semistable rank 2 vector bundles of degree $e$ on $X_{\text {red }}$. Then

(a) If $g \leq 2 \bar{g}-1$ (i.e. $\operatorname{deg}(\mathscr{N}) \geq 0$ ), it holds that $\mathrm{M}\left(\mathscr{O}_{X}, P_{d}\right)$ is equal to $\mathrm{M}(2, e)$.

(b) if $g>2 \bar{g}-1$ (i.e. if $\operatorname{deg}(\mathscr{N})<0$ ), all the irreducible components of $\mathrm{M}\left(\mathscr{O}_{X}, P_{d}\right)$ contain a stable generalized line bundle, except at most one, which is $\mathrm{M}(2, e)$, if it exists. If $\bar{g} \leq 1$, then $\mathrm{M}(2, e)$ is not an irreducible component of $\mathrm{M}\left(\mathscr{O}_{X}, P_{d}\right)$. On the other hand it is so if $\bar{g} \geq 2$ and $g \leq 4 \bar{g}-3$, i.e. $\operatorname{deg}(\mathscr{N}) \geq 2-2 \bar{g}$.

The first point of the fact is a restatement of [CK, Theorem 4.6] (observe that we have rescaled the irreducible components there treated to make more apparent what they are: in the notation of [CK] $\bar{Z}_{j}$ would be $\bar{Z}_{i}$ with $\left.i=[j / 2]+1\right)$. The second one is a reformulation of [CK, Theorem 4.7]. These results had already been stated, although without proof, in [DEL, Theorem 3.2(i)] in the particular case in which the normal bundle of $X_{\text {red }}$ in $X$, i.e. $\mathscr{N}^{-1}$ is the canonical line bundle of $X_{\text {red }}$. In the same article it was also pointed out that the analysis there developed holds in general for $\mathscr{N}^{-1}$ of sufficiently large degree.

It is time to state explicitly [CK, Question 4.8]:

Question 1. Assume that $\bar{g} \geq 2$ and $g>4 \bar{g}-3$, or equivalently $\operatorname{deg}(\mathscr{N})<2-2 \bar{g}$. Is $\mathrm{M}(2, e)$ an irreducible component of $\mathrm{M}\left(\mathscr{O}_{X}, P_{d}\right)$ ? 
The answer is negative. In order to prove that, it is sufficient to show that, under these hypotheses, the direct image of any semistable rank 2 vector bundle of degree $e$ on $X_{\text {red }}$ deforms to a generalized line bundle of degree $d$ on $X$. The following fact collects the ingredients of the proof.

Fact 2. Let $C$ be a smooth curve of genus $\bar{g}$ on $k$ and let $E$ be a rank 2 vector bundle of degree $e$ on it. Then the following hold:

(i) $s(E):=e-2 \max \{\operatorname{deg}(L) \mid L \subset E$ is a line bundle $\} \leq \bar{g}$; equivalently if $M$ is a maximal line subbundle (i.e. a line subbundle of maximal degree) of $E$ then $\operatorname{deg}(M) \geq(e-\bar{g}) / 2$. In particular if $E$ is semistable (resp. stable) then $(e-\bar{g}) / 2 \leq \operatorname{deg}(M) \leq e / 2$ (resp. $(e-\bar{g}) / 2 \leq \operatorname{deg}(M)<e / 2)$.

(ii) For a general semistable $E$, it holds that

$$
s(E)= \begin{cases}\bar{g}-1 & \text { if } e-\bar{g} \text { is odd } \\ \bar{g} & \text { otherwise. }\end{cases}
$$

In the second case, i.e. $s(E)=\bar{g}$, the maximal line subbundles of $E$ constitute a subscheme of dimension 1 in $\mathrm{Pic}^{(e-s(E)) / 2}(C)$.

(iii) Let $X$ be a ribbon such that $X_{\text {red }}=C$, let $\mathscr{N}$ be its nilradical and let $b$ a non-negative integer of the same parity of $d=e-$ $\operatorname{deg}(\mathscr{N})$. The direct image on $X$ of $E$ deforms to a generalized line bundle $\mathscr{I}$ of degree $d$ and index $b$ if and only if there exists a line bundle $L \subset E$ of degree $(e+\operatorname{deg}(\mathscr{N})+b) / 2$ and such that $\operatorname{Hom}\left((E / L) \otimes_{\mathscr{O}_{C}} \mathscr{N}, L\right) \neq\{0\}$.

The first point is equivalent to a classical result about ruled surfaces, that had already been proved by $\mathrm{C}$. Segre (obviously over $\mathbb{C}$, see $[\mathbf{S}]$ ) and had been rediscovered by M. Nagata (see [N]); it can be found in this form in [LN]. The first part of the second one is an immediate consequence of [LN, Proposition 3.1], while its second part is [LN, Corollary 4.7], although it has originally been proved in the context of ruled surfaces by M. Maruyama (see [M]). The third assertion is a restatement of [D, Théorème 7.2.3] (observe that in the cited article J.-M. Drézet works over $\mathbb{C}$, but his arguments can be applied to curves over any algebraically closed field).

The following theorem is the main result of the paper.

Theorem 1. Let $X$ be a ribbon of genus $g$ such that $\bar{g} \geq 2$, where $\bar{g}$ is the genus of $X_{\text {red. }}$. If $g \geq 4 \bar{g}-2$ (or equivalently $\operatorname{deg}(\mathscr{N}) \leq 1-2 \bar{g}$ ), then the direct image on $X$ of any rank 2 vector bundle $E$ of degree $e$ on $X_{\text {red }}$ deforms to a generalized line bundle $\mathscr{I}(h)$ on $X$ of degree $d=e-\operatorname{deg}(\mathscr{N})$ and of index $b_{E}(h)=-\operatorname{deg}(\mathscr{N})-s(E)-2 h$ for any integer $0 \leq h \leq[(-\operatorname{deg}(\mathscr{N})-s(E)) / 2]$. Moreover $E$ is (semi)stable if and only if $\mathscr{I}(0)$ is (semi)stable and these equivalent conditions imply that $\mathscr{I}(h)$ is stable for any $1 \leq h \leq[(-\operatorname{deg}(\mathscr{N})-s(E)) / 2]$. 
Proof. Let $E$ be a vector bundle as in the hypotheses, set $\delta=\operatorname{deg}(\mathscr{N})=$ $2 \bar{g}-g-1$ (so under our assumptions it is a negative integer), $s=s(E)$ and $b(h)=b_{E}(h)=-\delta-s-2 h$.

By Fact 2)(iii) the assertion is equivalent to the fact that $b(h)$ is non-negative and that there exists a line bundle $L(h) \subset E$ of degree $(e+\delta+b(h)) / 2$ such that $\operatorname{Hom}((E / L(h)) \otimes \mathscr{N}, L(h)) \neq\{0\}$.

Let us begin with $h=0$. Consider $M \subset E$ a maximal line subbundle; by Fact 2[(i) it holds that

$$
\operatorname{deg}(M)=\frac{e+\delta+b(0)}{2}=\frac{e-s(e)}{2} \geq \frac{e-\bar{g}}{2}>\frac{e+\delta}{2}
$$

where the last inequality holds because $g>4 \bar{g}-2$ is equivalent to $\delta<2-2 \bar{g} \leq-\bar{g}$ (recall that $\bar{g} \geq 2$ ). Hence $b(0)$ is a non-negative integer, as desired.

Thus it is sufficient to show that $\operatorname{Hom}((E / M) \otimes \mathscr{N}, M) \neq\{0\}$. It is immediate to verify that $M$ is a saturated subsheaf of $E$, being a maximal line subbundle, hence $E / M$ is a line bundle on $X_{\text {red }}$. So it holds that $\operatorname{Hom}((E / M) \otimes \mathscr{N}, M) \cong \mathrm{H}^{0}\left(X_{\text {red }},(E / M)^{-1} \otimes \mathscr{N}^{-1} \otimes M\right)$. The latter is surely nontrivial if $\operatorname{deg}\left((E / M)^{-1} \otimes \mathscr{N}^{-1} \otimes M\right)=-s-\delta \geq$ $\bar{g}$, by Riemann-Roch formula. This is true if either $g>4 \bar{g}-2$ (i.e. $\delta \leq-2 \bar{g}$ ) or $g=4 \bar{g}-2$ (i.e. $\delta=1-2 \bar{g}$ ) and $s<\bar{g}$. In the first case it holds that $-s-\delta \geq-\bar{g}-\delta \geq \bar{g}$, where the first inequality is due to Fact 2)(i), asserting that $s \leq \bar{g}$. In the second case it is immediate that $-s-\bar{\delta}>-\bar{g}-\delta \geq \bar{g}-1$.

By Fact [2(i), it remains only the case $\delta=1-2 \bar{g}$ and $s=\bar{g}$, in which $E$ is a general stable rank 2 vector bundle on $X_{\text {red }}$. Set $F(M)=$ $(E / M)^{-1} \otimes \mathscr{N}^{-1} \otimes M$. It holds that $\operatorname{deg}(F(M))=-s-\delta=\bar{g}-1$, so $h^{0}(F(M))>0$ if and only if $F(M)$ belongs to the theta-divisor of $\operatorname{Pic}^{\bar{g}-1}\left(X_{\text {red }}\right)$, which is ample. By the last assertion of Fact 2)(ii) there exists a 1-dimensional family of maximal line subbundles of $E$ and so the map $M \rightarrow F(M)$ defines a curve in $\operatorname{Pic}^{\bar{g}-1}\left(X_{\text {red }}\right)$ (by the fact $F(M) \otimes \mathscr{N}=M^{2} \otimes(\operatorname{det}(E))^{-1}$, hence $F(M) \neq F\left(M^{\prime}\right)$ for two generic maximal line subbundles $M$ and $M^{\prime}$ ). Thus by the ampleness of the theta-divisor the intersection of this curve with it is not zero; hence we can conclude that there exists at least one maximal line subbundle $M$ such that $F(M)$ belongs to it.

Now consider $1 \leq h \leq[(-\delta-s) / 2]$. By the previous part it holds that $b(0)=-\delta-s$ is non-negative, therefore also $b(h)=b(0)-2 h$ is non-negative.

Let $M$ be, as above, a maximal line subbundle of $E$ such that $\operatorname{Hom}((E / M) \otimes \mathscr{N}, M) \neq\{0\}$ (in other words such that $(E / M) \otimes \mathscr{N}$ can be seen as a line subbundle of $M$, because any non-trivial morphism between line bundles is injective) and let $L(h) \subset M$ a line subbundle of degree $(e+\delta+b(h)) / 2$. It is immediate to verify that the torsion-free part of $E /(L(h))$ is just $E / M$, hence $\operatorname{Hom}((E / L(h)) \otimes \mathscr{N}, L(h))=$ 
$\operatorname{Hom}((E / M) \otimes \mathscr{N}, L(h))$. It is clear that there exists an $L(h) \subset M$ such that $\operatorname{Hom}((E / M) \otimes \mathscr{N}, L(h))$ is not trivial if and only if there exists an $L(h) \subset M$ containing $(E / M) \otimes \mathscr{N}$; this holds if and only if

$$
\operatorname{deg}((E / M) \otimes \mathscr{N})=\frac{e+s+2 \delta}{2} \leq \operatorname{deg}(L(h))=\frac{e-s-2 h}{2},
$$

which is equivalent to $h \leq-\delta-s$. Requiring also that $b(h) \geq 0$ we find exactly $1 \leq h \leq[(-\delta-s) / 2]$. So for any integer $h$ in the desired range it is possible to find a line bundle $L(h) \subset M \subset E$ with the required properties.

The assertion about (semi)stability is almost trivial: $E$ is semistable (resp. stable) if and only if $s(E) \geq 0$ (resp. $>0$ ) if and only if $b(0)=-\delta-s \leq-\delta($ resp. $<-\delta)$ if and only if $\mathscr{I}(0)$ is semistable (resp. stable), where the first equivalence holds by definition and the last one is [CK, Lemma 3.2]. Moreover $b(h)<b(0)$ for any $1 \leq h \leq[(-\delta-s) / 2]$ hence $\mathscr{I}(h)$ is stable, if $b(0) \leq-\delta$, again by [CK, Lemma 3.2]. q.e.d.

Remark 1. It follows immediately from the proof and from Fact 2)(iii) that the estimate of the indices in the Theorem is sharp. Indeed, if there were an index $b>b(0)$ for which the theorem were true, one should have a line subbundle $L \subset E$ of degree strictly greater than that of a maximal line subbundle, which is a contradiction. On the other hand, if $b<b([(-\operatorname{deg}(\mathscr{N})-s(E)) / 2])$ and is of its same parity, $b$ is negative and so it cannot be the index of a generalized line bundle.

Corollary 1. If $\bar{g} \geq 2$ and $g \geq 4 \bar{g}-2$, then $\mathrm{M}(2, e)$ is not an irreducible component of $\mathrm{M}\left(\mathscr{O}_{X}, P_{d}\right)$. More precisely it is contained in $\bigcap_{\substack{j=0 \\ j \equiv m(2)}}^{m} \bar{Z}_{j}$, with $m=-\operatorname{deg}(\mathscr{N})-\bar{g}+1$ if $e-\bar{g}$ is odd and $m=-\operatorname{deg}(\mathscr{N})-\bar{g}$ if $e-\bar{g}$ is even. Moreover, it is not contained in any other irreducible component.

Proof. The hypotheses are the same of the Theorem, so it follows immediately from it and from Fact 2(ii) that $\mathrm{M}(2, e)$ is contained in $\bigcap_{\substack{j=0 \\ j \equiv m(2)}}^{m} \bar{Z}_{j}$.

The last assertion is a trivial consequence of the Remark. q.e.d.

\section{Acknowledgements.}

This paper is born as an aside to my doctoral thesis, which is in progress and is about the compactified Jacobian of a primitive multiple curve of multiplicity greater or equal than 3. I am grateful to my supervisor, Filippo Viviani, who introduced me to the articles [CK] and [D] and more generally to the subject and, moreover, gave me various suggestions about the exposition. I am also grateful to Edoardo Sernesi: my knowledge of one of the key ingredients of the proof, namely SegreNagata Theorem (i.e. Fact 2(i) is due to his unpublished notes about 
algebraic curves which he distributed confidentially in a preliminary version during a doctoral course about Brill-Noether theory. I would thank also the anonymous referee for his useful comments.

A.m.D.g.

\section{REFERENCES}

[BE] D. Bayer, D. Eisenbud, Ribbons and their canonical embeddings. Trans. Am. Math. Soc. 347 (3) (1995), 719-756.

[CK] D. Chen, J.L. Kass, Moduli of generalized line bundles on a ribbon. J. Pure Applied Algebra 220 (2016), 822-844.

[DEL] R. Donagi, L. Ein, R. Lazarsfeld, Nilpotent cones and sheaves on K3 surfaces, in: Birational Algebraic Geometry, Contemp. Math. 207, Amer. Math. Soc., Providence, RI, (1997), 51-61.

[D] J.-M. Drézet, Faisceaux cohérents sur le courbes multiples. Collect. Math. 57 (2) (2006), 121-171.

[LN] H. Lange, M.S. Narasimhan, Maximal subbundles of rank two vector bundles on curves, Math.Ann. 266 (1983), 55-72.

[M] M. Maruyama, On classification of ruled surfaces. Lectures in Mathematics. Kyoto Univ. No. 3, Tokyo (1970).

[N] M. Nagata, On self intersection number of vector bundles of rank 2 on a Riemann surface. Nagoya Math. J. 37 (1970), 191-196.

[S] C. Segre, Recherches générales sur les courbes et les surfaces réglés algébriques II. Math. Ann. 34 (1889), 1-25.

Dipartimento di Matematica e Fisica, Università Roma Tre, Largo San Leonardo Murialdo 1, 00146 Roma (ItAlia)

E-mail address: msavarese@mat.uniroma3.it 